Western University

Scholarship@Western

2013

\title{
Development of a 3D Ultrasound System to Investigate Post-Hemorrhagic Hydrocephalus in Pre-term Neonates
}

\author{
J. Kishimoto \\ Western University \\ D. Lee \\ Western University \\ K. St. Lawrence \\ Western University \\ W. Romano \\ Western University \\ A. Fenster \\ Western University \\ See next page for additional authors
}

Follow this and additional works at: https://ir.lib.uwo.ca/anatomypub

Part of the Anatomy Commons, and the Cell and Developmental Biology Commons

Citation of this paper:

Kishimoto, J.; Lee, D.; St. Lawrence, K.; Romano, W.; Fenster, A.; and de Ribaupierre, S., "Development of a 3D Ultrasound System to Investigate Post-Hemorrhagic Hydrocephalus in Pre-term Neonates" (2013). Anatomy and Cell Biology Publications. 32.

https://ir.lib.uwo.ca/anatomypub/32 
Authors

J. Kishimoto, D. Lee, K. St. Lawrence, W. Romano, A. Fenster, and S. de Ribaupierre 


\section{Development of a 3D ultrasound system to investigate post- hemorrhagic hydrocephalus in pre- term neonates}

J. Kishimoto

D. Lee

K. St. Lawrence

W. Romano

A. Fenster

S. de Ribaupierre 


\title{
Development of a 3D Ultrasound System to Investigate Post- Hemorrhagic Hydrocephalus in Pre-term Neonates
}

\author{
Kishimoto, $\mathrm{J}^{1,2,6}$, Lee, $\mathrm{D}^{3}$, St. Lawrence, $\mathrm{K}^{1,2}$, Romano, $\mathrm{W}^{5}$, Fenster, $\mathrm{A}^{2,6}$, de Ribaupierre, \\ $\mathrm{S}^{2,4}$ \\ ${ }^{1}$ Imaging Division, Lawson Health Research Institute, London, Ontario, Canada \\ ${ }^{2}$ Department of Medical Biophysics, The University of Western Ontario, London, \\ Ontario, Canada \\ ${ }^{3}$ Department of Neonatology, London Health Sciences Centre, London, Ontario, Canada \\ ${ }^{4}$ Department of Clinical Neurological Sciences, London Health Sciences Centre, London, \\ Ontario, Canada \\ ${ }^{5}$ Department of Diagnostic Radiology, London Health Sciences Centre, London, Ontario, \\ Canada \\ ${ }^{6}$ Imaging Research Laboratories, Robarts Research Institute, The University of Western \\ Ontario, London, Ontario, Canada
}

Keywords: 3D ultrasound, preterm neonates, intraventricular hemorrhage, post hemorrhagic hydrocephalus, bedside monitoring, cerebral ultrasound, transfontanelle

\begin{abstract}
Clinical intracranial ultrasound (US) is performed as a standard of care on neonates at risk of intraventricular hemorrhaging (IVH) and is also used after a diagnosis to monitor for potential ventricular dilation. However, it is difficult to estimate the volume of ventricles with 2D US due to their irregular shape. We developed a 3D US system to be used as an adjunct to a clinical system to investigate volumetric changes in the ventricles of neonates with IVH. Our system has been found have an error of within $1 \%$ of actual distance measurements in all three directions and volume measurements of manually segmented volumes from phantoms were not statistically significantly different from the actual values $(p>0.3)$. Interobserver volume measurements of the lateral ventricles in a patient with grade III IVH found no significant differences between measurements. There is the potential to use this system in IVH patients to monitor the progression of ventriculomegaly over time.
\end{abstract}

\section{INTRODUCTION}

Intraventricular hemorrhage (IVH) is a common problem among preterm infants with an occurrence of 12$20 \%$ in babies born at less than 35 weeks gestation, and at a rate of $45 \%$ in those born of extremely low birth weight (500-750g). ${ }^{1}$ Clinical intracranial ultrasound (US) is usually preformed within the first few days of life to diagnose IVH in at risk neonates. If detected, the grade of the hemorrhage is ranked clinically from I-IV (least to greatest severity). ${ }^{2}$ Neonates with IVH grade II or higher often develop an increase in ventricular size or ventriculomegaly. Often this dilation stabilizes over time, but in some cases can progress to hydrocephalus, which is associated with a greater risk of brain injury ${ }^{3}$ and is thought to be dependant on whether it is treated in a timely manner. This is why constant monitoring is required when interventions such as ventricular taps or drains might be necessary.

At present, neonates at risk of IVH are monitored by clinically with head circumference and by cerebral transfontanelle 2D ultrasound (US) for hemorrhage and potential ventricular dilation; however, the sensitivity of 2D US to detect dilation is poor, as it cannot accurately provide quantitative measures of irregular shapes such as the ventricles. Previous work ${ }^{4-9}$ on neonates have found 3D US to be feasible in a clinical setting. There is, however, a need to develop a system developed specifically to allow direct comparison between 2D and 3D US images from the same system.

\footnotetext{
Medical Imaging 2013: Ultrasonic Imaging, Tomography, and Therapy, edited by Johan G. Bosch, Marvin M. Doyley,
} Proc. of SPIE Vol. 8675, 86751M · C 2013 SPIE · CCC code: 1605-7422/13/\$18 · doi: 10.1117/12.2006646 
We have developed a portable 3D US system addition coupled to a conventional clinical 2D scanner (Phillips ATL HDI 5000) with a conventional curvilinear transducer (Phillips C8-5). This system is akin to the "tilted" scanner system described in Fenster et al. ${ }^{10}$ and is a modification from the breast system described in De Jean et al. ${ }^{11}$ Since this system acquires images using the same transducer as is used in clinical scans we are able to make direct comparisons to clinical images as well as the estimates from those images as to whether or not ventricular dilation is occurring.

In this paper we describe our new 3D US system as well as report on the validity of the volumetric measurements from 3D US images of phantom ventricles of dimensions and assess the system's ability to determine in vivo volumetric measurements of neonates.

\section{METHODOLOGY}

\subsection{D US System}

The system consists of a clinical 2D US transducer housed in a motorized attachment powered by a Faulhaber IE2400 1409 dc motor with a 416:1 gear ratio (MicroMo Electronics, Clearwater,FL), a MCDC controller for the motor, a Epiphan VGA2USB LR frame grabber (RB Computing, Nepean,ON) and a laptop computer. This 3D US scanner system can be used with any clinical US machine and with many different transducers. The system was housed on a small cart (see Fig. 1A), making it portable, which is essential for bedside measurement in the neonatal intensive care unit (NICU) where space is limited.

We used a Phillips ATL HDI 5000 US system and a Phillips C8-5 curved array 5-8 MHz broadband transducer for both phantom and patient scans.

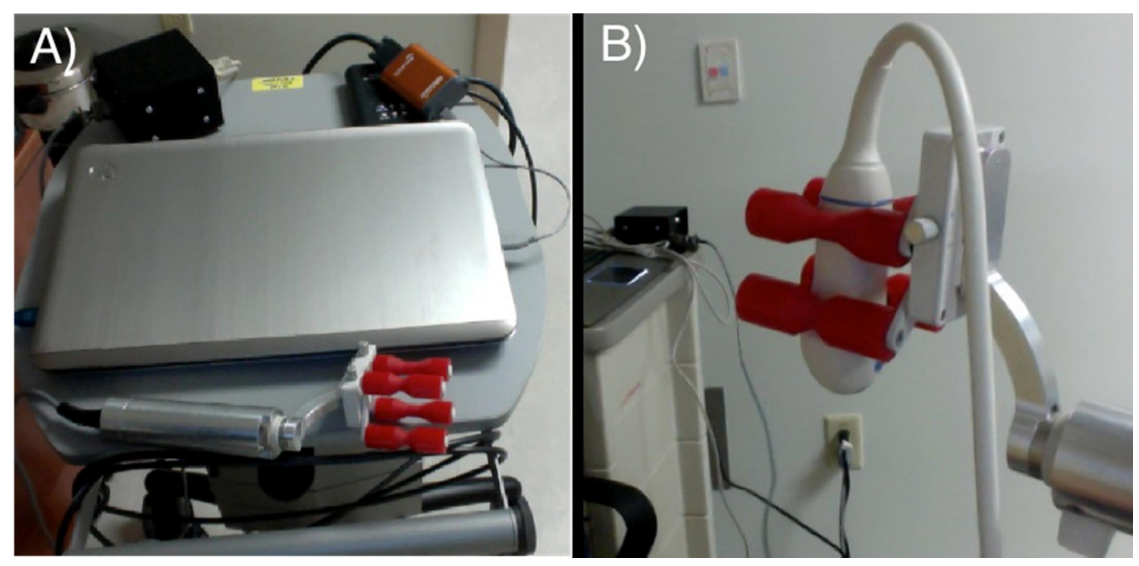

Figure 1. A) 3D imaging system including motor controller, frame grabber, laptop and motorized transducer housing. B) Transducer housing with attached C8-5 transducer

During image acquisition, the operator locates the middle of the target of interest, and then firmly holds onto the motor encasement while the device rotates the transducer on an axis at the probe tip. The transducer housing starts at 'centre', and once a scan begins to rotate the transducer to one angular extreme, which is when frames begin to be acquired, and commences once the transducer is rotated to the other extreme. The angular spacing between image frames can be between 0.2 and 0.3 degrees, and images are acquired at 25 frames/second with a total scan angle of 60 degrees for a total image acquisition time between 8-12 seconds.

To perform the 3D US scan, the motor controller, which is connected to the computer via a USB, tilts the transducer, while the frame grabber acquires 2D US images from the video output of the US machine throughout the scan. The software developed in our laboratory controls the motor's movements, acquires the 2D US images from the frame grabber, and reconstructs the 2D US images into a 3D US image as the 
images are acquired. ${ }^{10}$ This results in a 3D US image composed of a series of 2D US images spaced at a constant angular intervals arranged in a fan-like form.

\subsection{Geometric Validation of Reconstruction}

To ensure our reconstructed 3D US geometry is correctly calibrated, linear measurement in all three scanning directions (axial, lateral, and elevation) must be validated. To test the accuracy of these measurements, a phantom was scanned that consisted of strings spaced $1 \mathrm{~cm}$ apart in each direction in a solution of distilled water and $7 \%$ glycerol by weight (speed of sound $1540 \mathrm{~m} / \mathrm{s}$ ). ${ }^{11}$ The scanner was stabilized and the transducer face was completely submerged in the glycerol solution during image acquisition. The string phantom was then scanned at every calibrated depth $(4.9-16.0 \mathrm{~cm})$. The distance between strings was measured manually using our visualization software. Specifically, the distance between one intersection of a string to the next closest intersection was measured in the axial, lateral, or elevation direction four times per direction per depth. T-tests were performed between the measured data and the expected measurement of $1 \mathrm{~cm}$.

\subsection{Ventricle Volume Phantom - Accuracy Measurement}

Following the protocol approved by the Research Ethics Board at The University of Western Ontario (London, ON, Canada), T1 weighted MRI images (Ax FSPGR 3D, GE 1.5T) of a hydrocephalic infants with severe IVH were collected after the parents gave consent. The lateral ventricles were manually segmented in parallel slices. A positive model of the lateral ventricles (See Fig. 3a) was created using rapid prototyping (A FORTUS 400mc 3D Production System, Stratasys, Eden Prairie, MN) in ABS-M30i (acrylonitrile butadiene styrene). A negative mould of the ventricles was produced by casting the positive ventricle model in silicone SILBIONE® RTV 4420 A/B (Bluestar Silicone, East Brunswick, NJ) to create a two-part mould (Fig. 3b).

The ventricle-like phantom was made of an agar compound of 1L distilled water, 35g of agar powder (high strength A-6924, Sigma Chemicals, St Louis, MO) and 80mL glycerol (EMD Chemicals Inc. Gibbstown, $\mathrm{NJ}$ ). Brain-like medium was created using the $500 \mathrm{~mL}$ of this agar compound and $15 \mathrm{~g}$ of SigmaCell (Sigma Chemicals, St Louis, MO) to create soft-tissue mimicking scatter in US images. ${ }^{12}$

The phantom was also scanned using our 3D US system by locating the midline in the sagittal plane of the ventricle and scanning from one lateral extreme to the other. The images were acquired at a depth of $11.8 \mathrm{~cm}$, with a 60 degrees sweep, 0.3 degrees between frames, and at 25 frames per second.

The ventricles were manually segmented in parallel slices with $0.75 \mathrm{~mm}$ between slices through the sagittal plane. Two different observers performed the three measurements of the ventricles. The ventricle volume was compared to a gold standard of water displacement using t-test. ANOVA was performed to see if there was a difference between the two observers.

\subsection{In vivo Measurements}

Patients were recruited to the study if the first clinical US scan showed an IVH of at least grade II. Parents of the neonate were asked for their consent to follow their child in the study with a protocol approved by the Research Ethics Board at Western University (London, ON, Canada). Study data were collected following the routine cranial US exam. The clinical US transducer was placed in the motor housing and the 3D US volume was acquired. The US technician or a physician located the midline of lateral ventricles through the anterior fontanelle. Images were then acquired while the motor housing was held firmly and the probe tip is monitored to ensure constant contact while maintaining a low level of pressure applied to the fontanelle. Sagittal slices were acquired at a depth of $5-12 \mathrm{~cm}$ (usually $7.9 \mathrm{~cm}$ ) and were reconstructed in real-time into a 3D US image. Scans were performed with a 60 degree scan angle, a step size of 0.3 degrees at a frame rate of 25 frames/s. The scans were finished once an image of sufficient quality was acquired (ie. one with limited patient or operator movement, and the full ventricular volume). Generally, this was 
obtained in 1-4 3D US image acquisitions. The entire investigation, including routine US acquisition, lasted 10-25 minutes.

For the purposes of this paper, the first patient enrolled into the study was segmented 5 times to validate that manual segmentation could be replicated. A repeated measures analysis of variance (ANOVA) was performed on the volumes measured by two trained observers on different days with at least 24 hours in between the measures. The segmentation of the full ventricle volume took between 20-30 minutes.

\section{RESULTS}

\subsection{Geometric validation}
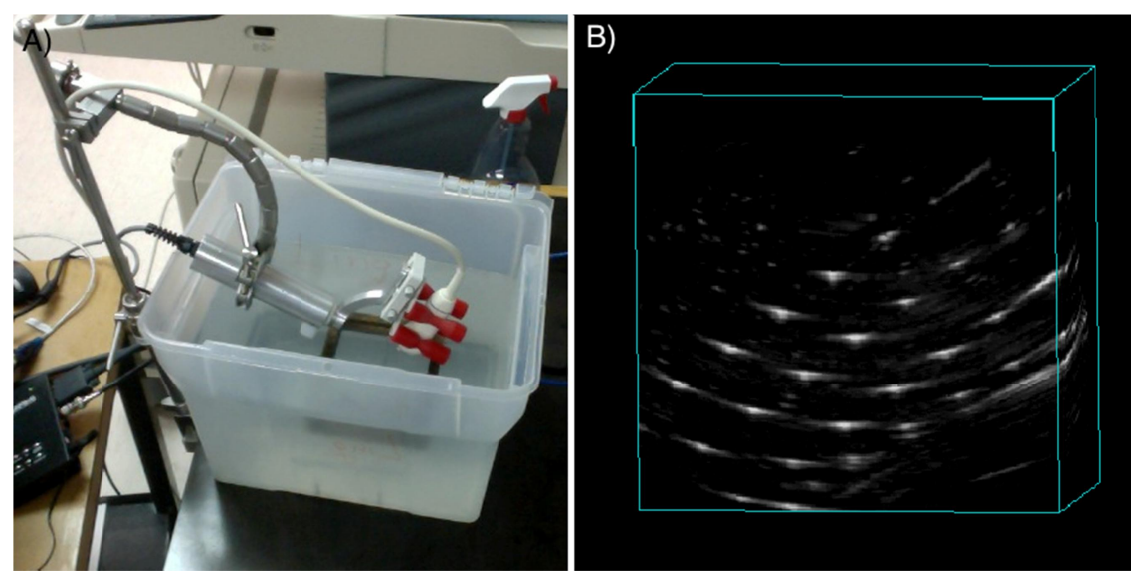

Figure 2A) String phantom set up for imaging. B) 3D US image of the string phantom at $7.9 \mathrm{~cm}$ depth.

Table 1. Manual distance measurements of the string phantom.

\begin{tabular}{cccc}
\hline Direction & Mean $(\mathbf{m m})$ & Stdev $(\mathbf{m m})$ & P-value \\
\hline Axial & 10.01 & 0.069 & 0.416 \\
Lateral & 10.02 & 0.084 & 0.185 \\
Elevation & 9.97 & 0.089 & 0.207 \\
\hline
\end{tabular}

Results of the performed t-tests failed to show a statistically significant difference from known value of 10 $\mathrm{mm}(\mathrm{p}<0.05)$.

\subsection{Volumetric Validation}

The mean volume measurement of the phantom was $276.9 \mathrm{~cm}^{3}$ for observer 1 and $274.7 \mathrm{~cm}^{3}$ for observer 2, which were not statistically significantly different from each other $(p=0.843)$. Both measurements were also found to be not statistically significantly different from the known value of $281 \mathrm{~cm}^{3}$ with $\mathrm{p}>0.3$ for both. 

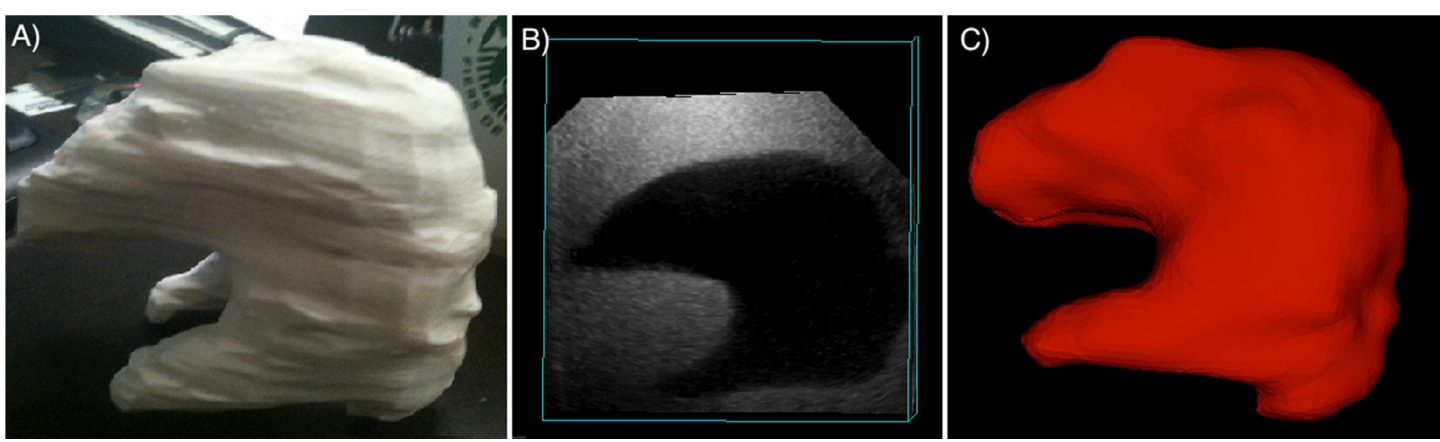

Figure 3A) Positive model of lateral ventricles created from patient MRI B) Saggital US image of agar phantom created from model C) Manually segmented surface of ventricles from 3D US.

\subsection{In Vivo Volume Assessment}

Table 2. Inter-observer volume measures of a neonate with bilateral grade III IVH

\begin{tabular}{ccc}
\hline Observer & Mean $\left(\mathrm{cm}^{3}\right)$ & Stdev $\left(\mathrm{cm}^{3}\right)$ \\
\hline 1 & 16.35 & 0.62 \\
2 & 17.33 & 1.67
\end{tabular}

No statistical significant difference was observed between the volume measurements $(\mathrm{p}=0.365)$, and most of the variance was between the observers (partial eta squared $=0.99$ ). The observed power was low $(0.125)$ meaning that although these measurements are encouraging, more patients are needed to be certain this finding was not an anomaly.
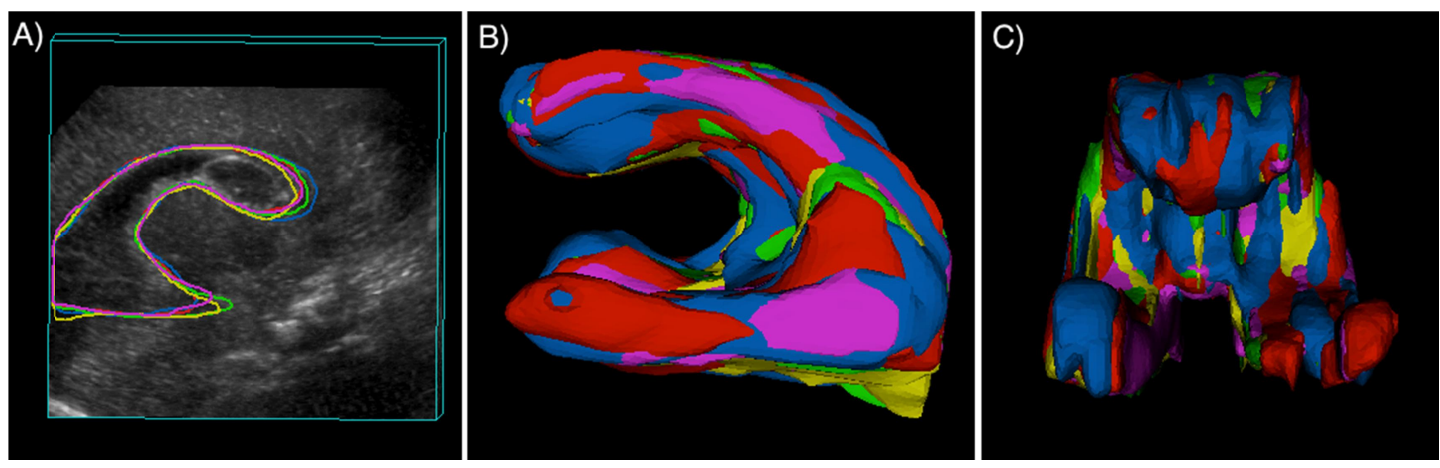

Figure 4A) Sagittal view of a patient scan with segmentation overlay. Each segmentation is done in a different colour. B) Sagittal view of segmentation surfaces. C) Coronal view of segmentation surfaces.

\section{DISCUSSION}

We have shown that our 3D US system can measure ventricle volumes accurately through the fontanelle of a neonate. Most importantly, we showed that even if it is performed using manual segmentation, lateral ventricular volumes could be measured precisely between different observers, for a patient with an abnormal ventricular system.

Much like the findings of Abdul-Khaliq $\mathrm{H}$ et al. ${ }^{5}$ our scanner should be easily transferable into a clinical setting. Whereas the system described in Abdul-Khaliq et al. rotated a 2D probe 180 around, our scanner tilts 60 degrees, and can acquire full ventricle volumes in a shorter time. Unfortunately, even though we have a portable system, due to the 'bulky' nature of the motorized transducer housing, not all neonates can 
be scanned in an enclosed incubator without having to adjust the patient's position, or opening up the incubator. As long as a clinician or nurse was available to adjust the patient's position more suitable for scanning, good images could be acquired. In neonates with very large ventricular systems, a scan angle of 60 degrees may not be enough to encompass the full ventricular volume. In such cases, the lateral ventricles will have to be imaged one at a time.

The bulkiness of the device can be addressed by the development of a miniaturized system more suitable to a neonatal incubator. The scan angle available to the technician should be increased so that neonates with larger ventricles can be scanned in one volume. Nevertheless, 3D US is faster than a conventional US examination, and provides the full volume of the ventricular system. In contrast, conventional 2D scans only provide limited information, and are highly technician dependent.

\section{CONCLUSION}

This 3D US system can reliably produce 3D US images of the neonatal ventricular system. There is the potential to use this system in IVH patients to monitor the progression of ventriculomegaly over time.

\section{REFERENCES}

[1] Wilson-Costello, D., Friedman, H., Minich, N. et al., "Improved survival rates with increased neurodevelopmental disability for extremely low birth weight infants in the 1990s," Pediatrics, 115(4), 997-1003 (2005).

[2] Papile, L. A., Burstein, J., Burstein, R. et al., "Incidence and evolution of subependymal and intraventricular hemorrhage: a study of infants with birth weights less than 1,500 gm," J Pediatr, 92(4), 529-34 (1978).

[3] Wilson-Costello, D., Borawski, E., Friedman, H. et al., "Perinatal correlates of cerebral palsy and other neurologic impairment among very low birth weight children," Pediatrics, $102(2 \mathrm{Pt} 1), 315$ 22 (1998).

[4] Salerno, C. C., Pretorius, D. H., Hilton, S. W. et al., "Three-dimensional ultrasonographic imaging of the neonatal brain in high-risk neonates: preliminary study," J Ultrasound Med, 19(8), 549-55 (2000).

[5] Abdul-Khaliq, H., Lange, P. E., and Vogel, M., "Feasibility of brain volumetric analysis and reconstruction of images by transfontanel three-dimensional ultrasound," J Neuroimaging, 10(3), 147-50 (2000).

[6] Riccabona, M., Nelson, T. R., Weitzer, C. et al., "Potential of three-dimensional ultrasound in neonatal and paediatric neurosonography," Eur Radiol, 13(9), 2082-93 (2003).

[7] Gilmore, J. H., Gerig, G., Specter, B. et al., "Infant cerebral ventricle volume: a comparison of 3D ultrasound and magnetic resonance imaging," Ultrasound Med Biol, 27(8), 1143-6 (2001).

[8] Haiden, N., Klebermass, K., Rucklinger, E. et al., "3-D ultrasonographic imaging of the cerebral ventricular system in very low birth weight infants," Ultrasound Med Biol, 31(1), 7-14 (2005).

[9] Nagdyman, N., Walka, M. M., Kampmann, W. et al., "3-D ultrasound quantification of neonatal cerebral ventricles in different head positions," Ultrasound Med Biol, 25(6), 895-900 (1999).

[10] Fenster, A., Downey, D. B., and Cardinal, H. N., "Three-dimensional ultrasound imaging," Urol Oncol, 46(5), R67-99 (2001).

[11] DeJean, P., Brackstone, M., and Fenster, A., "An intraoperative 3D ultrasound system for tumor margin determination in breast cancer surgery," Med Phys, 37(2), 564-70 (2010).

[12] Rickey, D. W., Picot, P. A., Christopher, D. A. et al., "A wall-less vessel phantom for Doppler ultrasound studies," Ultrasound Med Biol, 21(9), 1163-76 (1995). 